\title{
Note on Error Bounds for Numerical Integration
}

\author{
By J. H. Hetherington
}

\begin{abstract}
A length functional was defined in a previous publication which associates a length scale $r$ to every function analytic on a given interval. The ratio of $r$ to the mean panel size for numerical integration on that interval was defined as the sketchability. Stronger error bounds are given here for interpolative integration schemes in terms of the sketchability. Since these are not least upper bounds we also present lower limits for the least upper bounds obtained from actual numerical integrations of specific sketchability. We find the gap between the bounds and their lower limits to be small enough to be ignored for most applications.
\end{abstract}

1. Introduction. A length functional has been defined in [1], so that every function $f(x)$ analytic on the interval has length $r$ associated with it. $r$ is defined as the maximum value $r$ such that

$$
\left|f^{(n)}(x)\right| \leqq M r^{-n} n !
$$

for every value of $x$ in the interval and for all values of $n \geqq 0$, where $M$ is the maximum value of $|f(x)|$ on the interval. This length scale allows a definition of sketchability for a given numerical integration

$$
s=r \nu / H
$$

where $\nu$ is the number of panels and $H$ is the length of the interval of integration. An error ratio

$$
\mathcal{E}=|E| / H M,
$$

where $E$ is the integration error, was also defined in [1]. In [1], bounds on $\varepsilon$ for various quadrature schemes were found which are independent of whether quadrature rules are compounded, but depend on the sketchability. The bounds given were not least upper bounds (L.U.B.s). We have not succeeded in determining the L.U.B. on the error as a function of sketchability, but we have bracketed it rather closely. This has been done by first obtaining bounds which are much stronger for low sketchabilities and, secondly, finding error examples which are very close to the bounds so calculated.

2. Derivation of Stronger Bounds. The bounds presented in [1] were derived by application of the definition of length scale, Eq. (1), to the formula, valid for interpolative quadrature rules,

Received March 23, 1972, revised August 22, 1972.

AMS (MOS) subject classifications (1970). Primary 41A55, 41A25.

Key words and phrases. Newton-Cotes quadrature, Gaussian quadrature, quadrature error bounds.

Copyright @ 1973, American Mathematical Society 


$$
E \leqq G_{n} H^{n+1} F^{(n)},
$$

where $F^{(n)}$ is the maximum value of $\left|f^{(n)}(x)\right|$ for $x$ in the interval of integration. In [1] we only used the value $n$ equal to the order of the lowest order polynomial, which is not exactly integrated by the scheme. Equation (4), however, can be applied for any value $n$ equal to or less than that value; see for example [2]. We find these bounds, based on lower order derivatives, to be much stronger for lower sketchabilities.

The coefficients $G_{n}$ in Eq. (4) are given by

$$
G_{n}=\int_{-1 / 2}^{1 / 2}\left|K_{n}(x)\right| d x
$$

where $K_{n}(x)$ is the Peano kernel for the scheme for the $n$th derivative of the function and is defined by

$$
K_{n}(x)=E_{t}\left[(t-x)_{+}^{n-1}\right] /(n-1) !
$$

for $n \geqq 1$, where $E_{t}$ is the error functional for a unit interval. The case $n=0$ is singular, but the coefficient $G_{0}$ is given by

$$
G_{0}=\left[1+\sum_{i}\left|W_{i}\right|\right],
$$

where the weights $W_{i}$ of integration are normalized for the same interval, i.e., $\sum_{i} W_{i}=1$.

By combining Eqs. (1), (2), (3), and (4), error bounds can be written in the form

$$
\mathcal{E} \leqq B_{n} s^{-n}
$$

where the $B_{n}$ are

$$
B_{n}=\nu^{n} G_{n} n ! .
$$

We find that each member of this family of bounds is strongest for some range of $s$ for each quadrature scheme. Again, we find the $B_{n}$ and thus the bounds are independent of whether a rule is compounded or used singly, although we do not expect this to be true of the L.U.B.s. The bounds for $\varepsilon$ versus $s$ for a given quadrature consist of a series of straight line segments on a log-log plot.

Table I lists the quantities $G_{n}$ and $\log _{10} B_{n}$ for several Gaussian quadrature schemes and for several of the odd closed Newton-Cotes (NCC) quadratures. Also listed in Table I are the values of $\log _{10} s$ and $\log _{10} \varepsilon$ where the bounds intersect. On a plot of $\log _{10} \varepsilon$ versus $\log _{10} s$, the bound for a given quadrature scheme is obtained by connecting these intersection points by straight line segments. For $\log _{10} s$ greater than the last intersection listed in Table $I$, the bound is given by a straight line leaving that point with slope $-n$, where $n$ is the order of the highest order error term. For $\log _{10} s$ below the first intersection point, the $\log _{10} \mathcal{E}$ is bounded by a line of slope zero. Thus, $\log _{10} G_{0}=\log _{10} B_{0}$ can be found in Table I from $\log _{10} \varepsilon$ value of the intersection point for $n=1$. $G_{0}=2$ for all schemes with all positive weights.

3. Lower Limits on the Bounds. To obtain lower limits on the bounds, we have performed numerical integrations of known sketchability. The function 


$$
f(x)=A+\cos (\omega x)
$$

has been found useful. The length functional $r$ can be determined because the derivatives $f^{(n)}(x)$ with $F^{(n)} \leqq|\omega|^{n}$ are known. Thus, for a given $A$ and $\omega$, it is straightforward to apply Eq. (1) and obtain $r$, and thus the sketchability of the particular integration. By actually performing the numerical integration, the error ratio $\varepsilon=|E| / H M$ can also be determined since the exact integral is known. Thus, each integration yields a point on a $\log _{10} s$ versus $\log _{10} \varepsilon$ plot. The envelope of these points is a lower limit on the error bounds. Table II shows these lower limits for several values of sketchability for the same set of quadrature schemes as in Table I. We have included in Table II only uncompounded rules. Compounded rules have in cases calculated given smaller errors for a given sketchability. Because the function set of given sketchability is somewhat more restricted for a longer interval than for a short one, it would be expected that compounded rules would have slightly lower L.U.B.s. However, the integrals calculated indicate that the L.U.B.s are not very much lower and therefore the bounds reported here can be considered useful for compounded rules also.

TABLE I

The coefficients $G_{n}$ and $\log _{10} B_{n}$ and the coordinates $\log _{10} s$ and $\log _{10} \&$ of the intersection points of the bounds for various Gaussian and odd Newton-Cotes closed schemes.

\begin{tabular}{lccccc}
\hline \multicolumn{1}{c}{ Scheme } & $n$ & \multicolumn{1}{c}{$G_{n}$} & $\log _{10} B_{n}$ & $\log _{10} s$ & $\log _{10} \mathcal{E}$ \\
\hline 1 Gauss & 1 & .250 & -.602 & -.903 & .301 \\
(Midpoint) & 2 & .833 & -1.079 & -.477 & -.125 \\
2 Gauss & 1 & .127 & -.592 & -.893 & .301 \\
& 2 & $.101(-1)$ & -1.091 & -.499 & -.093 \\
& 3 & $.119(-2)$ & -1.240 & -.149 & -.793 \\
4 Gauss & 4 & $.231(-3)$ & -1.051 & .189 & -1.806 \\
& 1 & $.689(-1)$ & -.559 & -.860 & .301 \\
& 2 & $.273(-2)$ & -1.058 & -.499 & -.060 \\
& 3 & $.147(-3)$ & -1.249 & -.190 & -.678 \\
& 4 & $.842(-5)$ & -1.286 & -.037 & -1.136 \\
& 5 & $.544(-6)$ & -1.175 & .111 & -1.731 \\
& 6 & $.410(-7)$ & -.917 & .258 & -2.465 \\
& 7 & $.388(-8)$ & -.494 & .423 & -3.455 \\
& 8 & $.562(-9)$ & .172 & .665 & -5.156 \\
& 1 & $.363(-1)$ & -.537 & -.838 & .301 \\
& 2 & $.739(-3)$ & -1.024 & -.487 & -.050 \\
& 3 & $.199(-4)$ & -1.213 & -.189 & -.647 \\
& 4 & $.543(-6)$ & -1.273 & -.060 & -1.032 \\
& 5 & $.155(-7)$ & -1.214 & .059 & -1.509 \\
& 6 & $.460(-9)$ & -1.061 & .152 & -1.976 \\
& 7 & $.142(-10)$ & -.825 & .236 & -2.480 \\
& 8 & $.455(-12)$ & -.512 & .313 & -3.015 \\
& 9 & $.153(-13)$ & -.127 & .385 & -3.596 \\
& 10 & $.548(-15)$ & .330 & .456 & -4.234 \\
& 11 & $.210(-16)$ & .858 & .528 & -4.954 \\
& 12 & $.881(-18)$ & 1.462 & .604 & -5.790 \\
& 13 & $.414(-19)$ & 2.151 & .689 & -6.804 \\
& 14 & $.228(-20)$ & 2.941 & .790 & -8.119 \\
& 15 & $.159(-21)$ & 3.865 & .924 & -9.997 \\
& 16 & $.170(-22)$ & 5.000 & 1.135 & -13.153 \\
\hline
\end{tabular}


TABLE I (Continued)

\begin{tabular}{|c|c|c|c|c|c|}
\hline Scheme & $n$ & $G_{n}$ & $\log _{10} B_{n}$ & $\log _{10} s$ & $\log _{10} \varepsilon$ \\
\hline $\begin{array}{l}3 \text { NCC } \\
\text { (Simpson) }\end{array}$ & $\begin{array}{l}1 \\
2 \\
3 \\
4\end{array}$ & $\begin{array}{l}.139 \\
.123(-1) \\
.174(-2) \\
.347(-3)\end{array}$ & $\begin{array}{l}-.556 \\
-1.005 \\
-1.079 \\
-.875\end{array}$ & $\begin{array}{r}-.857 \\
-.449 \\
-.074 \\
.204\end{array}$ & $\begin{array}{r}.301 \\
-\quad .107 \\
-\quad .858 \\
-1.691\end{array}$ \\
\hline $5 \mathrm{NCC}$ & $\begin{array}{l}1 \\
2 \\
3 \\
4 \\
5 \\
6\end{array}$ & $\begin{array}{l}.738(-1) \\
.372(-2) \\
.280(-3) \\
.257(-4) \\
.289(-5) \\
.517(-6)\end{array}$ & $\begin{array}{r}-.530 \\
-.924 \\
-.968 \\
-.801 \\
-.449 \\
.183\end{array}$ & $\begin{array}{r}-.831 \\
-.394 \\
-.044 \\
.166 \\
.352 \\
.632\end{array}$ & $\begin{array}{r}.301 \\
-\quad .136 \\
-\quad .836 \\
-1.468 \\
-2.210 \\
-3.609\end{array}$ \\
\hline $7 \mathrm{NCC}$ & $\begin{array}{l}1 \\
2 \\
3 \\
4 \\
5 \\
6 \\
7 \\
8\end{array}$ & $\begin{array}{l}.627(-1) \\
.254(-2) \\
.140(-3) \\
.834(-5) \\
.559(-6) \\
.433(-7) \\
.426(-8) \\
.638(-9)\end{array}$ & $\begin{array}{r}-.424 \\
-.738 \\
-.740 \\
-. .586 \\
-.282 \\
.162 \\
.779 \\
1.635\end{array}$ & $\begin{array}{r}-.725 \\
-.313 \\
-.002 \\
.154 \\
.304 \\
.445 \\
.616 \\
.856\end{array}$ & $\begin{array}{r}.301 \\
-\quad .111 \\
-\quad .733 \\
-\quad 1.202 \\
-1.801 \\
-2.506 \\
-3.536 \\
-5.217\end{array}$ \\
\hline 9 NCC & $\begin{array}{r}1 \\
2 \\
3 \\
4 \\
5 \\
6 \\
7 \\
8 \\
9 \\
10\end{array}$ & $\begin{array}{l}.845(-1) \\
.273(-2) \\
.118(-3) \\
.525(-5) \\
.252(-6) \\
.131(-7) \\
.765(-9) \\
.521(-10) \\
.449(-11) \\
.589(-12)\end{array}$ & $\begin{array}{r}-.170 \\
-. .456 \\
-.441 \\
-.287 \\
-.005 \\
.394 \\
.908 \\
1.547 \\
2.340 \\
3.361\end{array}$ & $\begin{array}{r}-.559 \\
-.286 \\
.015 \\
.153 \\
.283 \\
.399 \\
.513 \\
.639 \\
.793 \\
1.021\end{array}$ & $\begin{array}{r}.389 \\
.116 \\
-\quad .486 \\
-\quad .901 \\
-\quad 1.419 \\
-1.999 \\
-2.685 \\
-3.567 \\
-4.797 \\
-6.850\end{array}$ \\
\hline
\end{tabular}

TABLE II

Maximum error ratios (as $\log _{10} \varepsilon$ ) found for the schemes for which bounds are given in Table I as computed from the integrals of the function $A+\cos (\omega x)$. Values omitted are in the region where integration of some polynomial gives error equal to the error bound in Table $\mathrm{I}$.

\begin{tabular}{rcccccccr}
\hline $\log _{10} s$ & $1 \mathrm{G}$ & $2 \mathrm{G}$ & $4 \mathrm{G}$ & $8 \mathrm{G}$ & $3 \mathrm{NCC}$ & $5 \mathrm{NCC}$ & $7 \mathrm{NCC}$ & 9NCC \\
\hline-.5 & -.298 & -.300 & -.288 &.- .315 & -.220 & -.285 & -.170 & .118 \\
-.4 & -.419 & -.410 & -.388 & -.415 & -.320 & -.437 & -.267 & .021 \\
-.3 & -.568 & -.600 & -.576 & -.583 & -.494 & -.537 & -.367 & -.079 \\
-.2 & -.737 & -.800 & -.776 & -.783 & -.694 & -.666 & -.467 & -.179 \\
-.1 & -.917 & -1.038 & -1.062 & -1.053 & -.911 & -.866 & -.663 & -.363 \\
.0 & -1.103 & -1.329 & -1.392 & -1.398 & -1.190 & -1.084 & -.863 & -.563 \\
.1 & - & -1.632 & -1.793 & -1.831 & -1.490 & -1.379 & -1.156 & -.854 \\
.2 & - & -1.966 & -2.280 & -2.375 & -1.811 & -1.714 & -1.492 & -1.195 \\
.3 & - & -2.325 & -2.826 & -3.033 & -2.162 & -2.109 & -1.897 & -1.612 \\
.4 & - & -2.699 & -3.433 & -3.837 & -2.532 & -2.551 & -2.389 & -2.119 \\
.5 & - & -3.082 & -4.101 & -4.777 & -2.912 & -3.046 & -2.943 & -2.715 \\
.6 & - & -3.470 & -4.805 & -5.855 & -3.298 & -3.562 & -3.554 & -3.400 \\
.7 & - & - & -5.541 & -7.063 & - & -4.110 & -4.228 & -4.163 \\
.8 & - & - & -6.301 & -8.377 & - & -4.678 & -4.934 & -4.989 \\
.9 & - & - & -7.076 & -9.780 & - & -5.256 & -5.673 & -5.871 \\
1.0 & - & - & -7.858 & -11.249 & - & -5.841 & -6.435 & -6.786 \\
\hline
\end{tabular}


When sketchability is high enough, a polynomial of the order of the highest error derivative can have its sketchability limited by the highest (constant) derivative. In this case, the error bound given is the L.U.B. and this occurs for sketchability $s \geqq$ $\nu n / 2$, where $\nu$ is the number of panels and $n$ is the order of the highest error term. In Table II, blanks have been left where the bound given in Table I can be shown to be an L.U.B. by this argument.

Fig. 1 shows the error bounds for the closed Newton-Cotes schemes on 3, 5, and 9 points. The shading of the lines in Fig. 1 indicates the range of values between the curves of Table I and Table II. For higher sketchabilities, the range is substantially narrower than the lines could be drawn. Fig. 2 is similar to Fig. 1, except for the Gaussian quadratures on 1, 2, 4, and 8 points. Here also, the width of the lines indicates the range of values between the curves of Table I and Table II.

4. Conclusions. It will be noted that, among the Newton-Cotes schemes for lower sketchabilities, we find stronger bounds for lower order rules while for high

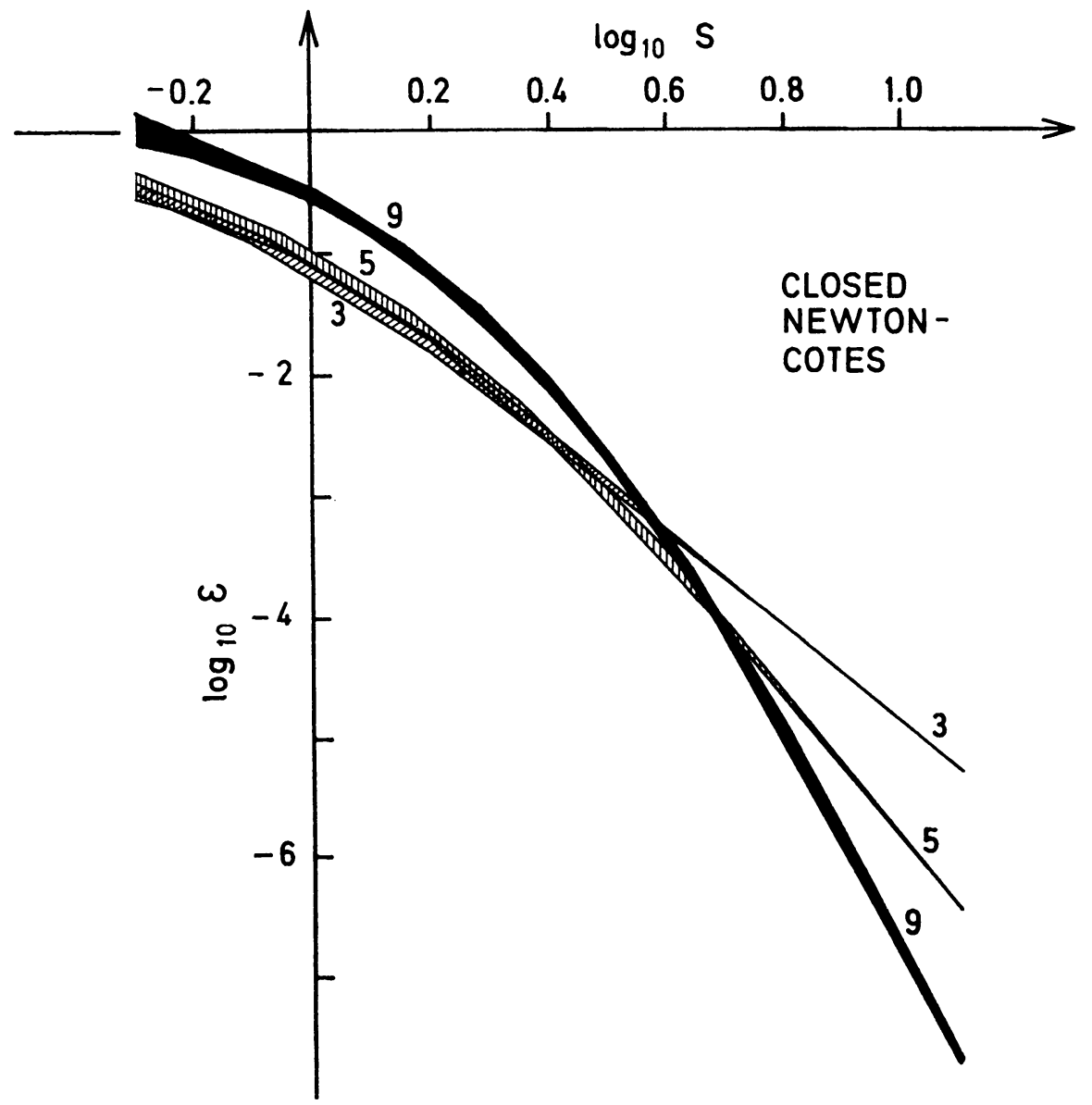

FIGURE 1

Log-log plot of the error bounds versus sketchability for the Newton-Cotes 3, 5, and 9-point closed quadrature schemes. Shading shows the gap between the bounds and actual integrations which have been performed. 
sketchabilities we find stronger bounds for high order rules. The possible range of the least bounds is small enough that these intersections exist for the least bounds. For the Gaussian quadratures, the bounds also probably intersect, but they are so close to each other that it is more convenient to think of them as being coincident at low sketchability but spreading out at higher sketchabilities. In any case, the ranges in which the least bounds are here bracketed for Gaussian quadratures are completely overlapping at low sketchability.

Application of these bounds to the function of the form

$$
\sum_{i} A_{i} /\left(a_{i}^{2}+\left(x-b_{i}\right)^{2}\right),
$$

where the $a_{i}$ and $b_{i}$ are real and the $A_{i}$ are real positive, is particularly simple because the length functional $r$ is just the shortest distance between the points $a_{i}+i b_{i}$ in the complex $x$-plane and the interval of integration.

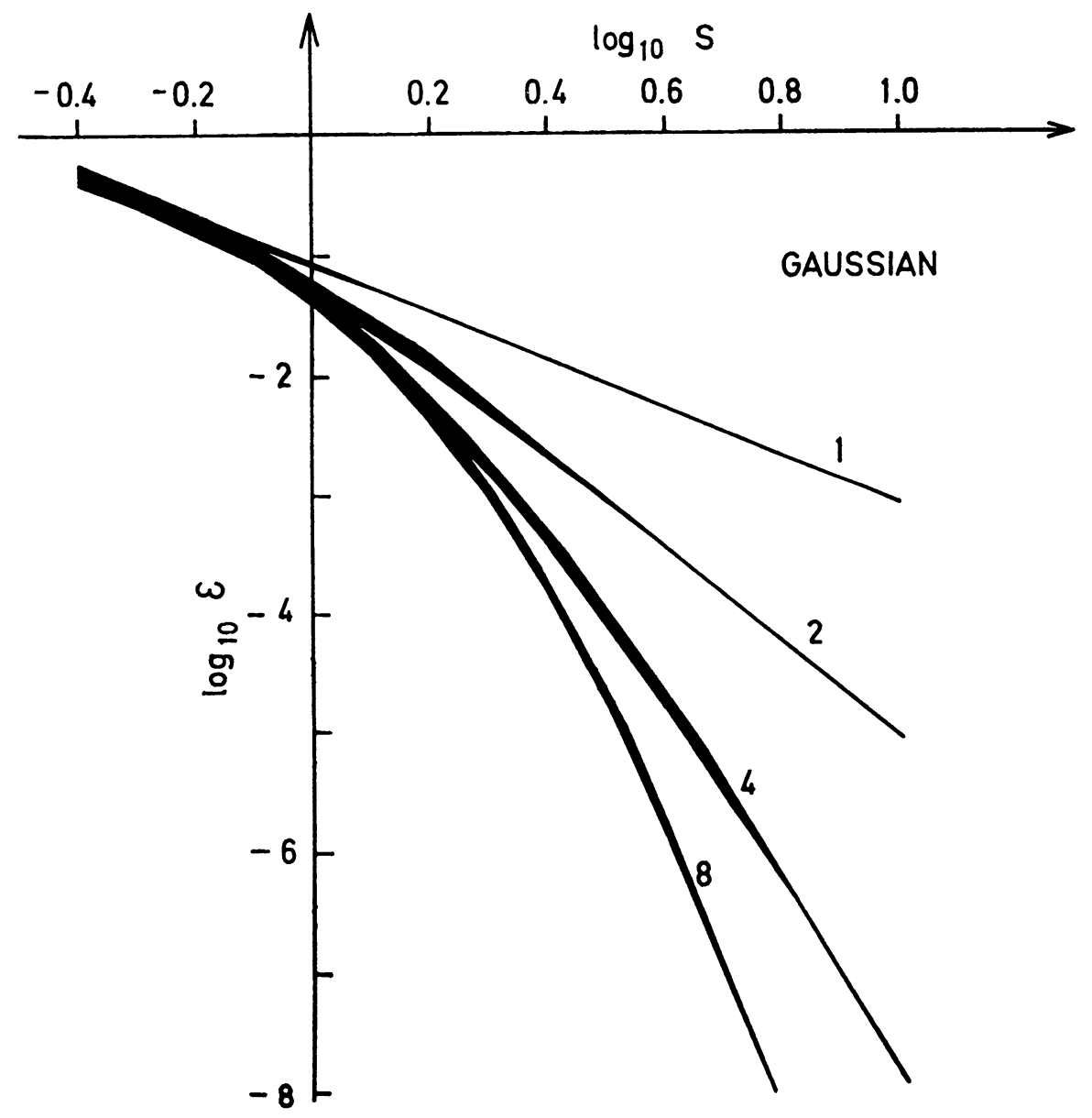

FIGURE 2

Log-log plot of the error bounds versus sketchability for the Gaussian 1, 2, 4, and 8-point schemes. The gap between the bounds and actual integrations is indicated by the width of the lines. 
In other cases, the length functional may sometimes be determined without too much difficulty from knowledge of the derivatives, for example, the function $A+$ $\cos (\omega x)$ which we have used above.

Because the quantity $r$ is less than the distance to the nearest singularity, it may be possible from a knowledge of the analytic structure of the function to put an upper limit on $r$ and thus on the sketchability of a given integration.

For integrations by the Newton-Cotes schemes, we find that when a certain fractional accuracy is desired, it can most efficiently be obtained from the quadrature scheme for which the bound gives the lowest sketchability for the desired accuracy. For example, if it is desired to obtain between about 2.5 and 4.0 digits of accuracy with one of the three Newton-Cotes schemes shown in Fig. 1, the 5-point scheme or its compounds would be the most efficient. For accuracies below 2.5 digits, the 3-point scheme or its compounds would seem to be better, while for accuracies above 4.0 digits, one finds the 9-point scheme or its compounds to be definitely more efficient. This result is independent of the value of $r$ for the function to be integrated.

5. Applications. Consider the integration

$$
I=\int_{-6}^{6} 1 /\left(1+x^{2}\right) d x \sim \pi
$$

The theory can be used to decide how to numerically integrate this integral to obtain a desired error bound. We will seek methods which yield a bound $\mathcal{E} \leqq 10^{-2}$ that is in terms of fractional error as a fraction of the answer $(|E| / I) \sim(12 / \pi) \times 10^{-2}$. We will consider integrating this in three ways: First, a single quadrature over the whole interval; second, by using a compounded formula; third, by breaking the interval nonuniformly and using different rules in different intervals.

Consider the possibility of using a single NCC rule over the whole interval. Because of the form of the integrand, we may apply the rule stated in Section 4. We find $r=1$ and therefore, for an $n$-point NCC quadrature, we obtain $s=(n-1) / 12$.

If we desire $\varepsilon \leqq 10^{-2}$, then we examine Fig. 1 and find that, for $s \gtrsim 2$, 9-point NCC gives sufficient accuracy. However, if $s=2$, we must have $n=25$ which is a very large order for Newton-Cotes. Returning to Fig. 1, we imagine that such higher order NCC quadratures need still higher $s$ for the accuracy desired and we cannot prove that by using higher and higher order NCC quadratures the numerical integration will converge.

On the other hand, a single high order Gaussian rule could be expected, from Fig. 2 , to require $s \simeq 1.6$ for $\mathcal{E} \leqq 10^{-2}$, even for a very high order quadrature. Therefore, we find that, with a 20-point Gaussian quadrature, we would have the desired accuracy. Experimentally, we obtain $\log _{10} \varepsilon=-3.23$ for 20 -point Gaussian.

Next, consider Simpson's rule, i.e., the compounded 3-point NCC rule. We find that in each subinterval $r \geqq 1$, and the length of the subinterval is $H=24 /(n-1)$, where $n$ is the total number of points on the whole interval $[-6,6]$. The bound on the actual error in each subinterval is therefore

$$
|E| \leqq \mathcal{E} H M \leqq \mathcal{E} \cdot 24 /(n-1)
$$


since $M \leqq 1$ on each subinterval. We may define $\mathcal{E}_{\text {whole }}$ as

$$
\mathcal{E}_{\text {whole }} \leqq\left(\sum\left|E_{\text {single }}\right|\right) / M H
$$

where $M$ and $H$ refer to the interval $[-6,6]$ and substituting, we find

$$
\mathcal{E}_{\text {whole }} \leqq \frac{n-1}{2} \cdot \mathcal{E} \cdot \frac{24}{n-1} /(1 \cdot 12)=\mathcal{E} .
$$

But $s \geqq(n-1) / 12$ for each subinterval. We find from Fig. 1 that on each subinterval the limit $\varepsilon \leqq 10^{-2}$ for $s=1.8$.

Therefore, we expect $\mathcal{E}_{\text {whole }} \leqq 10^{-2}$ for about 23 points. We obtain experimentally $\log _{10} \varepsilon=-3.25$ for 23-point Simpson's rule.

Had we compounded Gaussian quadratures, we would have proceeded similarly except that, because the necessary sketchability is lower $(\simeq 1.6)$, we find that a total of about 20 points are needed independently of the order of the Gaussian rules used, provided they are 4-point or higher.

Finally, we may break the interval into irregularly sized subintervals. Since the singularities in the complex plane are nearest the point $x=0$ on the real axis, we need the points to be concentrated in that region in order to make the sketchability more uniform. Furthermore, since the maximum $M$ in subintervals further from $x=0$ is less, we should in fact allow $s$ to be less in those regions if we wish the error per unit length $|E| / H$ to be made approximately uniform. For simplicity, we may try to hold $s$ approximately uniform and apply 3-point NCC on each irregular subinterval. For $\varepsilon \leqq 10^{-2}$, we need $s \simeq 1.8$ as determined from Fig. 1. Therefore, we can take subintervals $[0,1],[1,2.5]$ and $[2.5,6]$ and their reflections because with 3-point NCC each of these subintervals has $s=2,1.9,1.5$, respectively. We have a total of 13 points on the whole interval, but we expect $\varepsilon \lesssim 10^{-2}$, a rather more efficient integration than any of the above examples. Experimentally, we obtain $\log _{10} \varepsilon=-3.48$.

We may, in the analysis of an integral, want to know the values $r$ and $M$ for a function which is the product of two functions for which $r$ and $M$ are known. It is easy to bound $r$ for a function which differs at most by a constant from the product of two such functions. Suppose $g(x)=f_{1}(x) f_{2}(x)$ on $\left[x_{1}, x_{2}\right]$ and suppose $r\left\{f_{i}(r)\right\}=r_{i}$ and $M\left\{f_{i}(x)\right\}=M_{i}$; then the coefficients of the power series for $f_{1}$ and $f_{2}$ are bounded so that, if

$$
f_{i}(x)=\sum_{n=0}^{\infty} a_{n}^{i}\left(x-x_{0}\right)^{n}
$$

for $x_{0} \in\left[x_{1}, x_{2}\right]$, then $\left|a_{n}^{i}\right| \leqq M_{i} r_{i}^{-n}$.

Considering the product of the power series, we have

$$
g(x)=\sum_{n=0}^{\infty} A_{n}\left(x-x_{0}\right)^{n}
$$

where

$$
A_{n}=\sum_{m=0}^{n} a_{m}^{1} a_{n-m}^{2}
$$

From the bounds we have on $a_{n}^{i}$, we obtain 


$$
\left|A_{n}\right| \leqq M_{1} M_{2} \sum_{m=0}^{n} r_{1}^{-m} r_{2}^{-n+m}
$$

Assuming without loss of generality that $r_{2} \leqq r_{1}$, we have

$$
\left|A_{n}\right| \leqq M_{1} M_{2} r_{2}^{-n} \sum_{m=0}^{n}\left(r_{2} / r_{1}\right)^{m}
$$

Therefore, there exist functions $g(x)+C_{n}$ which have $M$ and $r$ bounded by

$$
\begin{aligned}
r & \geqq\left(1-\left(r_{2} / r_{1}\right)^{n+1}\right)\left(1-\left(r_{2} / r_{1}\right)^{n+2}\right)^{-1} r_{2}, \\
M & =\left(1-\left(r_{2} / r_{1}\right)^{n+1}\right)^{n+1}\left(1-\left(r_{2} / r_{1}\right)^{n+2}\right)^{-n} M_{1} M_{2} .
\end{aligned}
$$

We may simplify these bounds by writing for $n=0,1, \cdots$, and without specifying the size of $r_{1}$ relative to $r_{2}$,

$$
\begin{aligned}
r & \geqq \frac{(n+1)}{(n+2)} \min \left(r_{1}, r_{2}\right), \\
M & =\frac{n+2}{2} M_{1} M_{2},
\end{aligned}
$$

and

$$
\begin{aligned}
r & \geqq \min \left(r_{1}, r_{2}\right), \\
M & =\frac{\max \left(r_{1}, r_{2}\right)}{\left|r_{1}-r_{2}\right|} M_{1} M_{2} .
\end{aligned}
$$

Since the addition of the constant to $g(x)$ will not affect the absolute error of any integration scheme, the theory we have given can still be applied to the function $g(x)$. However, because a constant added to the function will affect the fractional error as defined by $\mathcal{E}=|E| / H M$, we will have to take account of it in determining fractional error.

We may now consider the integral

$$
\int_{-1}^{1} \frac{\cos (\omega x)}{\left(x-z_{1}\right)\left(x-z_{2}\right)} d x
$$

where $z_{1}$ and $z_{2}$ are complex parameters and where $\omega$ is a real parameter. An integral of this character, when integrated numerically, might need a lot of points or a few depending on the values of the parameters. We can define a sufficiency condition for the subdivision of this integral. On any subinterval of $[-1,1]$, the functions $f_{i}=\left(x-z_{i}\right)^{-1}$ have $r_{i}=r\left\{f_{i}\right\}$ equal to the distance in the complex plane from the point $z_{i}$ to the nearest point in the subinterval. And $M\left\{f_{i}\right\}=\left(r_{i}\right)^{-1}$. Also, $r\{\cos (\omega x)\}$ $\geqq 1 /|\omega|$ and $M\{\cos (\omega x)\}=1$.

As a result of our above considerations, we know that the integrand of (12) plus some constant is a function which on any subinterval has

$$
r \geqq \alpha \min \left(1 /|\omega|, r_{1}, r_{2}\right)
$$

and $M=\beta M_{1} M_{2}$. Without any analysis of which of the family of bounds (10) is best, we select the case $n=3$ and obtain 


$$
\begin{aligned}
r & \geqq\left(\frac{4}{5}\right)^{2} \min \left(1 /|\omega|, r_{1}, r_{2}\right), \\
M & =(6.25) M_{1} M_{2} .
\end{aligned}
$$

Let us suppose that we desire the result of the integration to about 6 digits relative to the integral of the absolute value of the integrand. (We measure the fractional error this way because it is convenient because of the considerable cancellation which can occur in (12).) Because of the factor 6.25 in $M$, we need to require $\varepsilon$ to be about $(6.25)^{-1} \times 10^{-6}$ on each subinterval in order to achieve this accuracy. From Fig. 2 or Table I, we can determine that, for 8-point Gaussian, the most efficient rule we have tabulated in this accuracy range, a sketchability of about 5 is required. Therefore, the length $H$ of the subintervals should be bounded by

$$
H \leqq \frac{8}{5}\left(\frac{4}{5}\right)^{2} \min \left(\frac{1}{|\omega|}, r_{1}, r_{2}\right) \leqq r \nu / s .
$$

Equation (13) is a sufficiency condition on $H$ such that the fractional accuracy will be about $10^{-6}$. This prescription is simple enough and specific enough for the programming of a computer to make the subdivision automatically on the basis of the parameters $\omega, z_{1}, z_{2}$.

Thus, if $z_{1}$ (or $z_{2}$ ) lies very close to the integration interval, subdivision will be finer in that neighborhood than in parts of the interval further from $z_{1}$ (or $z_{2}$ ). Also, the form (13) will prevent $H$ from being too large to adequately treat oscillations due to $\cos (\omega x)$ when $|\omega|$ is large.

Physics Department

Michigan State University

East Lansing, Michigan 48823

1. J. H. Hetherington, "An error bound for quadratures," Math. Comp., v. 26, 1972, pp. 695-698.

2. P. J. Davis \& P. Rabinowitz, Numerical Integration, Blaisdell, Waltham, Mass., 1967. Section 4.3. MR $35 \# 2482$. 